\title{
Application of Different Simulation Strategies for the Analysis of Multi- Stroke Localised-Incremental Forging Operations
}

\author{
A.M. Camacho ${ }^{a}$, M.M. Marín ${ }^{b}$, E.M. Rubio ${ }^{c}$ and M.A. Sebastián ${ }^{d}$ \\ Department of Manufacturing Engineering, National Distance University of Spain (UNED), Spain \\ aamcamacho@ind.uned.es, 'bmmarin@ind.uned.es, cerubio@ind.uned.es, \\ dmsebastian@ind.uned.es
}

Keywords: Localised-incremental forging, FEA, Implicit, Explicit, ALE, mass scaling.

\begin{abstract}
The analysis of LIF operations is often difficult, especially when several strokes are necessary to obtain the final shape of the part. The use of FE models is highly recommended and it is known that implicit FE models give more robust results than explicit FE models; however, with the former methodology there are several problems that must be faced. With the main goal to extend the analysis capability of LIF operations, some explicit FE models are developed in this paper and results are compared to those obtained by implicit methodology. It can be concluded that taking into account these guidelines, the developed models could be further used in the analysis of multistrokes LIF operations, where more complicated trajectories are going to be used.
\end{abstract}

\section{Introduction}

Flexibility, lower forces compared to die-defined forming and good formability of the finished parts are some of the advantages in the use of incremental forming, as highlighted in the paper by Groche et al. [1]; however, the authors also indicated that these technologies have some limitations that must be overcome mainly by an advanced design. Simulations by the Finite Element Method play an important role to cope this challenge, and new simulation strategies and methodologies are being developed by researchers in metal forming field. An example is the paper of Hirt et al. [2], where a multi-mesh method designed ad hoc is implemented for its use in incremental bulk metal forming processes such as open die forging and ring rolling. This method uses a FE mesh which is fine in the deformation zone and coarse in the remaining areas. A second mesh, fine over the entire volume, is used to store computed values and to minimize the loss of accuracy. Meanwhile, the numerical simulation of Localised-Incremental Forging operations is a challenging task. The analysis of LIF operations is often difficult, especially when several strokes are necessary to obtain the final shape of the part. The use of Finite Element models is mandatory because incremental operations throughout a workpiece are a hard task to analyse with conventional analytical methods. It is known that, in general, implicit FE models give more robust results than explicit FE models; however, with the former methodology there are several problems that must be faced. Incremental forming simulation is still highly time consuming: as concluded by Bambach et al. [3], in one study about single point incremental sheet forming, implicit simulations require much more simulation time, mainly due to the required iterations which are used in solvers such as ABAQUS/Standard to achieve well-defined contact conditions. On the other hand, different convergence problems such as, for example, those related to contact non linearities must often be solved. In the scientific literature there exist several attempts to overcome this kind of problems [4]. The first works realised to analyse LIF operations used an implicit methodology, as it was shown in the paper of Camacho et al. [5], where simple strokes where simulated. In this work different numerical strategies are tested in order to choose the best ones for the efficient analysis of a multi-stroke LIF operation. With the main goal to extend the analysis capability of LIF operations, some explicit FE models are developed in this paper and results are compared to those obtained by implicit methodology. 


\section{Methodology}

Approach to the problem. The problem consists of a two-strokes LIF operation where a simple punch of square section is forming the workpiece, applying a reduction in every stroke of $10 \%$. The dimensions and geometry of the workpiece are presented in Fig. 1 (a). The displacement of the workpiece is constrained laterally and an overlapping of $B / 2$ is considered between strokes, being $B$ the side-length of the punch section.

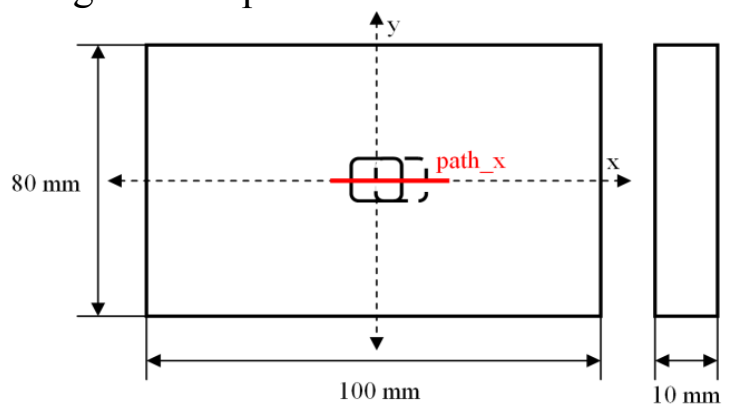

(a)

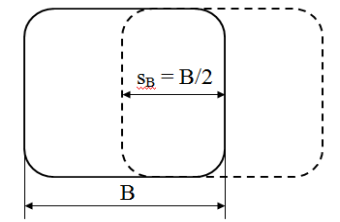

(b)

Fig. 1. (a) Dimensions of the workpiece; (b) Punch section and definition of "overlapping"

The material of the workpiece is a paraffin wax used typically in physical modeling of metal forming processes. Its flow curve is shown in Fig. 2, where a softening behavior is observed. The FE code Abaqus [3] is chosen for the analysis. A first model is analysed by using the implicit solver (Abaqus/Standard); afterwards, the explicit solver (Abaqus/Explicit) is chosen in different models, combining the following analysis strategies: adaptive mesh domain-ALE, refinement of the mesh and mass scaling. These strategies are used in order to choose the best models for further LIF analysis where more complicated trajectories of the punch can be required. Geometrical results, forces, residual stresses and strains are compared throughout the paper.

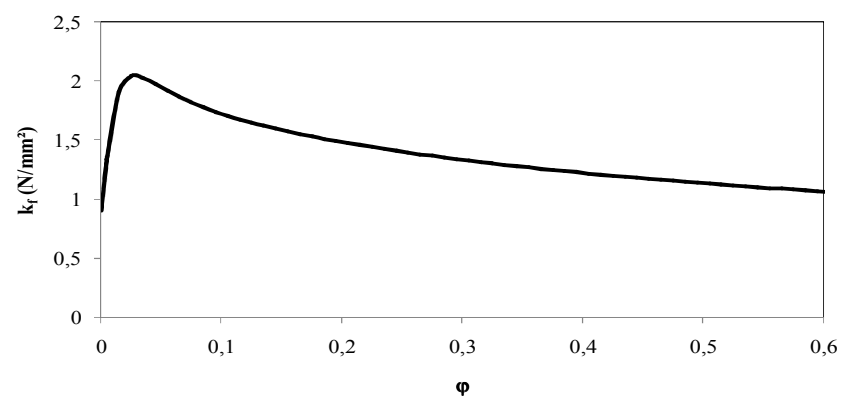

Fig. 2. Flow curve of the paraffin at room temperature (true stress versus true plastic strain)

Implicit model. The implicit model will be analysed by the solver Abaqus/Standard and it will be named "Model-0" from now on. As described in the Abaqus User's Manual [6], to solve for equilibrium in non linear problems, Abaqus/Standard uses an incremental-iterative solution based on the Newton-Raphson technique. Five "static/general" steps are defined in this model in order to specify the displacements of the punch, either vertically and horizontally. The mesh element used is C3D8R, corresponding to a 8 node linear brick with reduced integration and hourglass control. Some partitions are included in the mesh in order to define a finer mesh in the deformation zone and a coarser one in the rest of the workpiece.

Explicit model. The explicit dynamics analysis procedure is based upon the implementation of an explicit central-difference integration rule. But the key to the computational efficiency of the explicit procedure is the use of diagonal element mass matrices because the accelerations at the beginning of the increment are computed by:

$\ddot{u}_{i}=(M)^{-1}\left(P_{i}-I_{i}\right)$ 
The explicit procedure requires no iterations and no tangent stiffness matrix. Based on these fundamentals, a first explicit model is created. The mesh element is taken from the explicit element library, and it is also the C3D8R element. Three "dynamic/explicit" steps are defined: the first and the third for the definition of the vertical punch displacement and the second for the horizontal translation of the punch to the next position. For the application of the displacements in every step, two amplitudes are modeled, as shown in Fig. 3. As showed in the figure, a time period of 0.02 is defined for the $1^{\text {st }}$ and $3^{\text {rd }}$ step, and 0.005 for the $2^{\text {nd }}$ one.

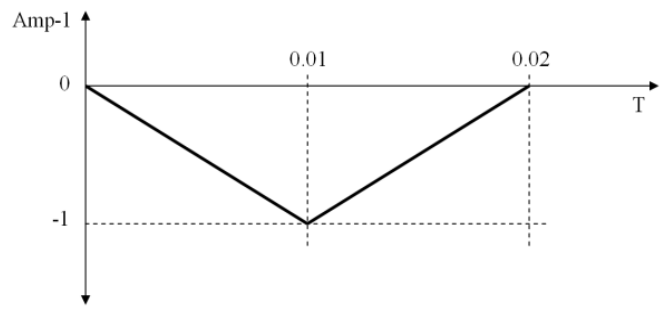

(a)

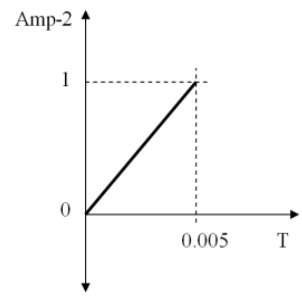

(b)

Fig. 3. Definition of step amplitudes in Abaqus/Explicit

Because the nature of metal forming problems is quasi-static, the simulation of this kind of problems with explicit dynamics is computationally impractical when the process is modeled in its natural time period. One solution to reduce the number of increments required is using the so called "mass scaling", that allows to model metal forming processes in their natural time scale by artificially increasing the material density by a factor of $f^{2}$, which increases the "stable time increment" by a factor of $f$. In order to calculate the solution in a reasonable time, the first explicit model ("Model-1") considers a mass scaling factor of 9. Another interesting capability of Abaqus/Explicit is the successful implementation of the Arbitrary Lagrangian-Eulerian (ALE) adaptive meshing; with this technique, the mesh motion is constrained to the material motion only at free boundaries [6]. This way the mesh distortion is minimized by adjusting mesh within the material free boundaries. ALE adaptive meshing uses a single mesh definition that is gradually smoothed within analysis steps. This is specially recommended when multiple strokes are considered in a LIF process, particularly when incremental forming strategies are considered; so this is the motivation of including the Model-2, where the ALE technique is implemented in all the steps of the analysis in order to reduce the mesh distortion during the forming processes. Finally, a fourth model is developed ("Model-3") with ALE and refinement of the mesh in the forming area; in this case, a mass scaling factor of 64 is also included to achieve a faster convergence. A detail of the meshes used can be found in Fig. 4.

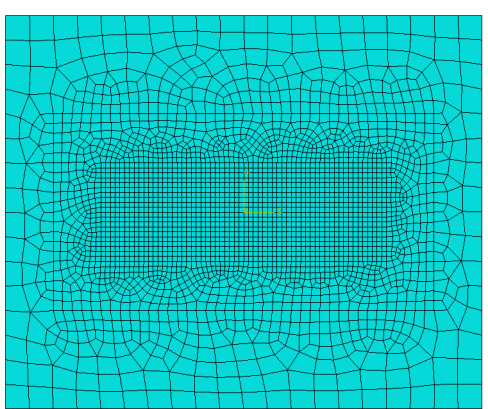

(a)

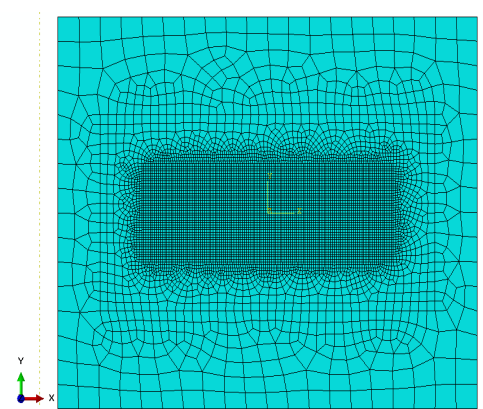

(b)

Fig. 4. Final mesh of (a) "Model-0", "Model-1" and "Model-2"; (b) Model-3 
A summary of the FE models developed can be found in Table 1.

Table 1. FE Models

\begin{tabular}{|c|c|c|c|c|}
\hline Model & $\begin{array}{c}\text { Numerical } \\
\text { methodology }\end{array}$ & Mass scaling & ALE & Mesh refinement \\
\hline Model-0 & Implicit & No & No & No \\
\hline Model-1 & Explicit & Yes (9) & No & No \\
\hline Model-2 & Explicit & Yes (9) & Yes & No \\
\hline Model-3 & Explicit & Yes (64) & Yes & Yes \\
\hline
\end{tabular}

Output variables. Forces, the final geometry of the groove and the residual stresses and strains are going to be analysed: punch reaction force in the $\mathrm{z}$ direction (RF), vertical displacement of the nodes along the center line in the $X$ axis (path_x, Figure 1), equivalent residual stresses along path_x (s, von Mises) and equivalent residual strains along path_x (PEEQ)

\section{Results}

Justification. When more than two strokes are considered, the implicit model (Model-0) is not able to complete the analysis and the simulation takes a long time due to the explained limitations of the implicit methodology. Because of this, it is necessary to find solutions that let us improve the convergence in the simulation of LIF operations. For this purpose, some explicit models are developed and the results in forces, geometry of the groove and residual stresses and strains are compared in order to define the best strategy in every case.

Forces. The results in forces for the second stroke are presented in Fig. 5. In this figure the trend of the forces for the four models can also be observed.

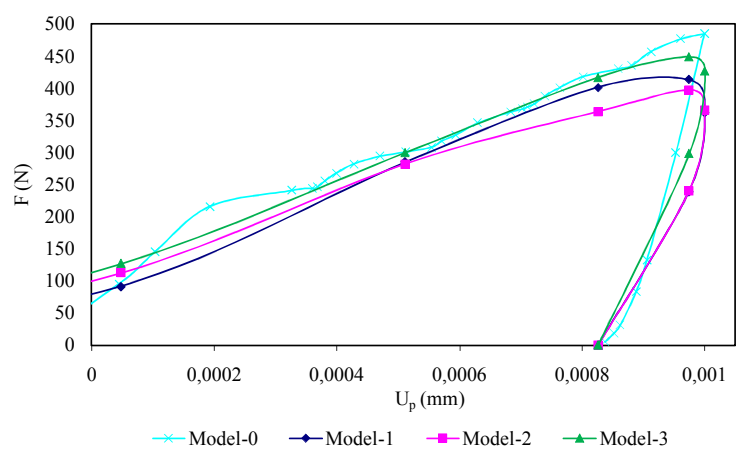

Fig. 5. Forces in the 2nd stroke

Geometry of the groove. Another interesting matter to be analysed is the determination of the final shape after forming. Fig. 6 represents the final geometry of the groove after the two strokes for the four FE models. In this case, the groove is registered when the punch has been totally removed from the contact surface. In general, results in the base of the groove are quite similar and all the models show a small peak as a result of the second forming stage. The highest differences are encountered again for the Model-1, especially at the final contact edge, where a high peak is observed compared to the one for Model-0. Model-2 gives better results, although Model-3 shows again the best results. 


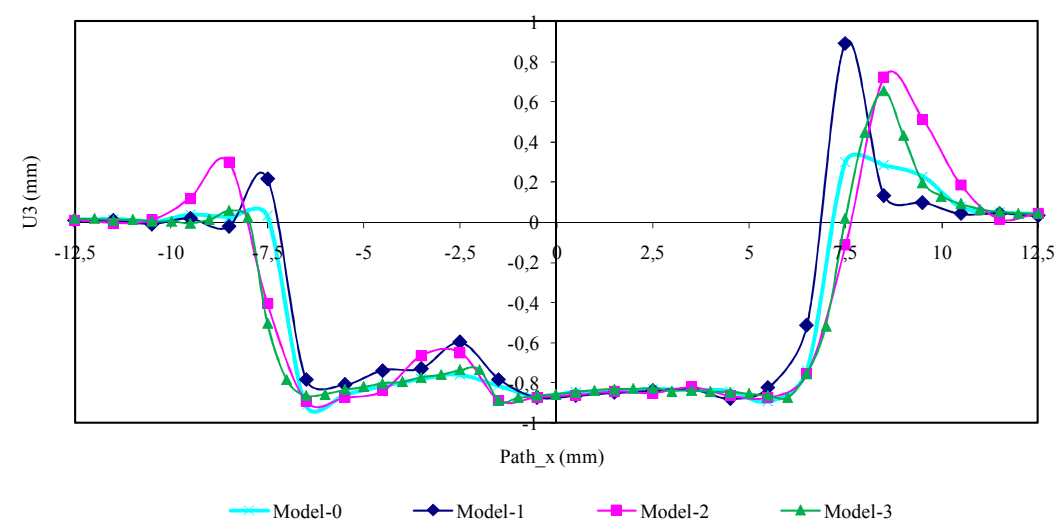

Fig. 6. Final shape of the groove after the forming process

Residual stresses. When residual stresses are evaluated, Fig. 7 must be analysed. In this case, the Arbitrary Lagrangian-Eulerian adaptive meshing is not recommended when trying to study the behavior of stresses, due to the differences found in Model-2. It seems to be that the way the mesh is improved has a high influence on the stress distributions, due to the fact that the integration points, where stresses are calculated, seems to be directly affected by this adaptive meshing. Arbitrary Lagrangian Eulerian (ALE) adaptive meshing provides control of mesh distortion [6], but accuracy is not guaranteed. So it is necessary to pay attention to this result, when evaluating residual stresses with this model. Mesh refinement in Model-3 is again a good strategy to improve the accuracy of the results. However, in this case Model-1 provides the best results.

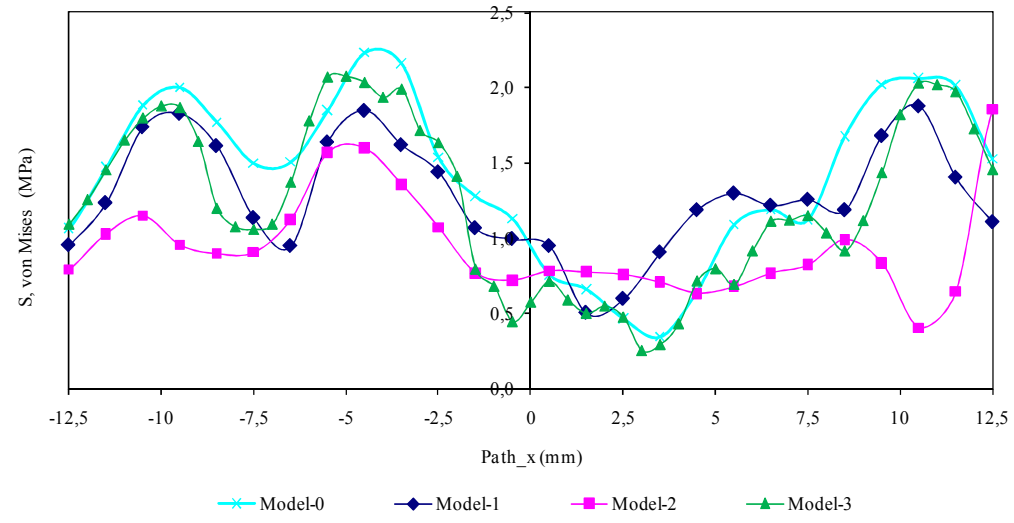

Fig. 7. Equivalent residual stresses along the surface

Residual strains. Equivalent residual strains are also registered in Fig. 8. A similar analysis to the residual stresses can be done when comparing all the different models.

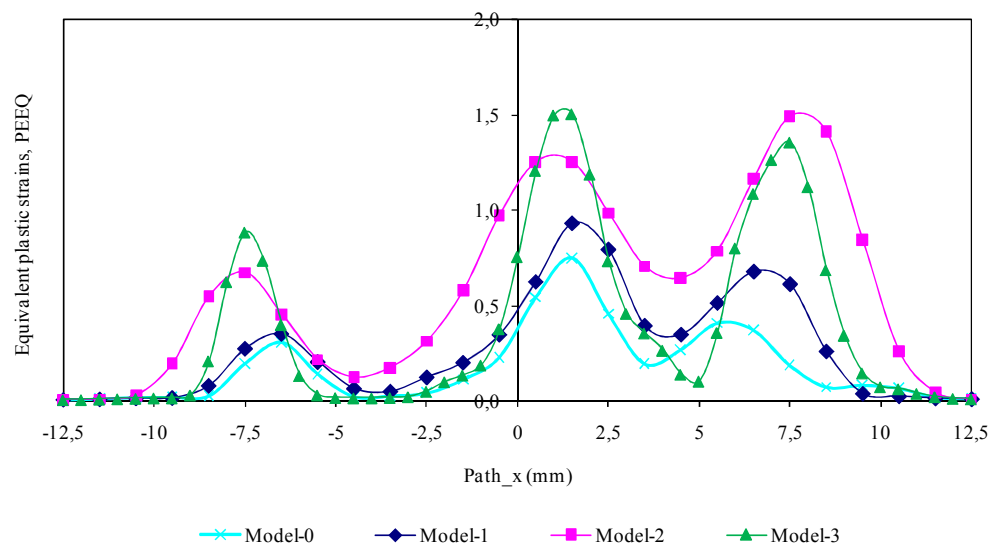

Fig. 8. Equivalent residual strains along the surface 
In this case, again, the best results are obtained for the Model-1, and the worst ones for the Model-2. Model-3 shows improved results when compared to Model-2 ones, but the differences in the three "peaks" are also relevant. The same explanation than for residual strains can be done when discussing and justifying these results.

\section{Conclusions}

In this paper, different strategies for the efficient simulation of LIF processes have been used. Using an implicit model as a reference of comparison, results in forces, final geometry and residual strains and stresses have been compared for three explicit models. The main results are summarised in Table 2, where the best (B) and the worst (W) models are indicated, depending on the variable of interest.

Table 2. Summary (B: best model; W: worst model)

\begin{tabular}{|c|c|c|c|c|}
\hline Model & Forces & Geometry & $\begin{array}{c}\text { Residual } \\
\text { stresses }\end{array}$ & $\begin{array}{c}\text { Residual } \\
\text { strains }\end{array}$ \\
\hline Model-1 & W & W & B & B \\
\hline Model-2 & & & W & W \\
\hline Model-3 & B & B & & \\
\hline
\end{tabular}

As a general conclusion it can be said that taking into account these guidelines, the developed models could be further used in the analysis of multi-strokes LIF operations, where more complicated trajectories will be required.

\section{Acknowledgements}

The authors gratefully acknowledge to the Institute of Metal Forming of the RWTH Aachen University, especially Prof. Hirt and Dr. Bambach, for all the kind supports, and the Spain's Ministry of Science and Innovation for funding this work via Project DPI2009-07300 and the Spain's Ministry of Education via National Human Resources Mobility Programme (Grant "José Castillejo") of the National R\&D\&I Plan 2008-2011.

\section{References}

[1] P. Groche, D. Fritsche, E.A. Tekkaya, J.M. Allwood, G. Hirt and R. Neugebauer: CIRP Ann. Manuf. Techn. Vol. 56 (2007), p. 2.

[2] G. Hirt, R. Kopp, O. Hofmann, M. Franzke and G. Barton: CIRP Ann. - Manuf. Techn. Vol. 56 (2007), p. 1.

[3] M. Bambach, G. Hirt, J. Ames, M. Azaouzi, J.L. Batoz and L. Campagne, in: Initial experimental and numerical investigations into a class of new strategies for single point incremental sheet forming (SPIF), edited by D. Banabic, Proceedings of ESAFORM 2005, The Romanian Academy Publishing House Bucharest (2005).

[4] A.E. Tekkaya: The Arab. J. for Sci. and Eng. Vol. 30 (2005), p. 1C.

[5] A.M. Camacho, C. Vallellano, F.J. García-Lomas and M.A. Sebastián: Steel Res. Int. Vol. 81 (2010), p. 9.

[6] D.Hibbitt, B. Karlsson and P. Sorensen, in: ABAQUS v6.10, User's Manuals (2010). 